Research Article

\title{
The Probable Association between Chronic Toxoplasma gondii Infection and Type 1 and Type 2 Diabetes Mellitus: A Case-Control Study
}

\author{
Shahrzad Soltani (D), ${ }^{1}$ Sanaz Tavakoli $\left(\mathbb{D},{ }^{2}\right.$ Mohamad Sabaghan $\left(\mathbb{D},{ }^{3}\right.$ Mehdi Sagha Kahvaz (iD), \\ Marzieh Pashmforosh (D), ${ }^{3}$ and Masoud Foroutan $\left.{ }^{1}\right)^{1}$ \\ ${ }^{1}$ USERN Office, Abadan Faculty of Medical Sciences, Abadan, Iran \\ ${ }^{2}$ Department of Parasitology and Mycology, School of Medicine, Isfahan University of Medical Sciences, Isfahan, Iran \\ ${ }^{3}$ Behbahan Faculty of Medical Sciences, Behbahan, Iran
}

Correspondence should be addressed to Marzieh Pashmforosh; marzie_pf@yahoo.com and Masoud Foroutan; masoud_foroutan_rad@yahoo.com

Received 19 April 2021; Revised 6 May 2021; Accepted 13 May 2021; Published 25 May 2021

Academic Editor: Massimiliano Lanzafame

Copyright (c) 2021 Shahrzad Soltani et al. This is an open access article distributed under the Creative Commons Attribution License, which permits unrestricted use, distribution, and reproduction in any medium, provided the original work is properly cited.

\begin{abstract}
Purpose. The probable association between Toxoplasma gondii (T. gondii) infection and diabetes mellitus (DM) is still controversial, and there are several studies with conflicting results. Thus, this study was performed to assess the possible association between chronic T. gondii infection and type 1 diabetes mellitus (T1DM) and T2DM. Methods. In this case-control study, a total of 105 diabetic subjects including 36 patients with T1DM and 69 patients with T2DM were recruited. In addition, 150 nondiabetic subjects were enrolled as controls. Each case group had its own control group. Each participant completed a structured questionnaire obtaining demographic information. Serum samples were examined for T. gondii-specific IgG antibody using enzyme-linked immunosorbent assay (ELISA) method. Results. Analysis revealed that $69.4 \%$ and $34.0 \%$ of patients with T1DM and control subjects were serologically positive for T. gondii, respectively (odds ratio (OR): 4.41; $95 \%$ confidence interval (CI): $1.75-11.06 ; P=0.001$ ). Moreover, $72.5 \%$ of T2DM patients and $29.0 \%$ of healthy individuals were seropositive for T. gondii (OR: 6.44; 95\% CI: 3.25-12.74; $P<0.001$ ). Among risk factors, only contact with cats was significantly associated with IgG seroprevalence in both T2DM patients $(P<0.001)$ and control subjects $(P=0.045)$. Conclusion. Although the results showed that chronic T. gondii infection is significantly associated with T1DM and T2DM, there remain many questions regarding the exact mechanisms of $T$. gondii in the pathogenesis of DM.
\end{abstract}

\section{Introduction}

Toxoplasma gondii (T. gondii) is an obligate apicomplexan intracellular parasite that is capable of infecting nearly all warm-blooded animal species, including humans [1]. There are various routes of $T$. gondii transmission to humans: ingestion of oocyst-contaminated food or water, eating cystinfected raw meat, vertical transmission from mother to fetus, organ transplantation, and blood transfusion [2-5]. It is estimated that one-third of the human population worldwide are infected with this parasite $[2,6,7]$. Previous systematic review articles in Iran have reported high
T. gondii seroprevalence rates of more than $45 \%$ in various human groups, including HIV/AIDS patients, cancer patients, transplant recipients, and hemodialysis patients when compared to lower seroprevalence rates observed in the general population including healthy blood donors and pregnant women $[8,9]$.

Diabetes mellitus (DM) is one of the major worldwide public health concerns of the 21 st century. It is estimated that the number of persons suffering from DM will increase to 552 million $(7.7 \%)$ in 2030 [10]. Diabetic patients have suppressed immune systems, potentially indicating that these subjects may be more susceptible to acquire $T$. gondii 
[11-13]. Type 1 diabetes mellitus (T1DM) is characterized by hyperglycemia due to the deficiencies in insulin hormone release, while type 2 diabetes mellitus (T2DM) is hallmarked by the failure to properly respond to insulin [10].

Since previous studies on the possible association between T. gondii infection and DM have reported conflicting results $[11,13]$, we decided to conduct a case-control investigation to shed light on the probable association between chronic T. gondii infection and T1DM and T2DM.

\section{Materials and Methods}

2.1. Study Area. The study was carried out in Khorramshahr city (Khuzestan province, southwest Iran, $30.4256^{\circ} \mathrm{N}$, $48.1891^{\circ} \mathrm{E}$ ) (Figure 1). At the 2016 census, its population was 170,976 . Khorramshahr city has hot summers (up to $55^{\circ} \mathrm{C}$ ) and cold winters $\left(1^{\circ} \mathrm{C}\right)$. The annual rainfall is around $140 \mathrm{~mm}$.

2.2. Study Design and Sample Collection. In this case-control study, a total of 105 cases including 36 patients with T1DM and 69 patients with T2DM were recruited from Valiasr Hospital (affiliated to the Abadan Faculty of Medical Sciences) from December 2019 to March 2020. A total of 150 control subjects were also enrolled. Each case group had its own control group. In the diabetic groups, inclusion criteria were as follows: fasting plasma glucose greater than or equal to $7.0 \mathrm{mmol} / \mathrm{L}$ and/or 2-hour plasma glucose greater than or equal to $11.1 \mathrm{mmol} / \mathrm{L}$ [14]. Healthy individuals were defined as control group if they had no previous history of diagnosis of diabetes and had fasting and 2-hour glucose measures under the common thresholds for diabetes. The diabetic patients in both case groups with metabolic disorders and those receiving immunosuppressive drugs were excluded from the current research.

2.3. Questionnaire. Each participant completed a structured questionnaire which obtained the following demographic information: age, gender, residence, education level, contact with cat, source of drinking water, and consumption of raw or undercooked meat.

2.4. Serological Assay. All the patients and control subjects had $5 \mathrm{~mL}$ of venous blood drawn. The samples were then centrifuged at $1700 \times \mathrm{g}$ for 5 minutes and kept at $-20^{\circ} \mathrm{C}$ till tested. In order to detect anti-T. gondii IgG antibody titer in the sera, a commercially available (Torch-IgG, Trinity Biotech Company) enzyme-linked immunosorbent assay (ELISA) kit was used according to the manufacturer's instructions.

2.5. Statistical Analysis. All data were imported into the Statistical Package for the Social Sciences (SPSS) software (version 21) (SPSS Inc., Chicago, IL, USA) for analysis. Chisquare and Fisher's exact tests were used to compare the variables. The significance level was defined to be less than $0.05(P<0.05)$.

\section{Results}

3.1. Seroepidemiology of T. gondii Infection in T1DM Patients. The seroprevalence of $T$. gondii infection in T1DM and control subjects was estimated to be $69.4 \%$ (25/36) and $34.0 \%$ (17/50), respectively, which showed a statistically significant difference (odds ratio (OR): $4.41 ; 95 \%$ confidence interval (CI): $1.75-11.06 ; P=0.001)$. Demographic characteristics of patients with T1DM and nondiabetic subjects, such as age group, gender, residence, education level, source of drinking water, and consumption of raw/undercooked meat, are presented in Table 1. T1DM patients in the age group of 21-30 years (80.0\%) showed the highest seroprevalence. No significant difference was observed between females $(82.35 \%)$ and males $(57.89 \%)$ of T1DM patient group $(P=0.109)$. T1DM patients who lived in rural areas (81.81\%) had higher seroprevalence of $T$. gondii than those who were in urban regions $(64.0 \%)$, but no statistically significant difference was observed $(P=0.254)$. In addition, the seroprevalence of T. gondii infection in T1DM patients with different education levels was not significantly different $(P=0.261)$. $T$. gondii seroprevalence was not significantly different among T1DM patients with the history of contact with cats $(P=0.073)$, source of drinking water $(P=0.571)$, and consumption of raw/undercooked meat $(P=0.609)$ (Table 1).

3.2. Seroepidemiology of T. gondii Infection in T2DM Patients. T2DM patients $(72.5 \%)$ showed a higher seroprevalence of T. gondii infection than nondiabetic group (29.0\%) (OR: 6.44; 95\% CI: 3.25-12.74; $P<0.001)$. T1DM patients in the age group of more than 60 years showed the highest rate of infection with T. gondii (73.91\%). No significant difference was observed between males (67.64\%) and females $(77.14 \%)$ in T2DM patients $(P=0.377)$. About $74.41 \%$ of T2DM patients living in urban areas were seropositive for T. gondii, while in rural regions $69.23 \%$ were found to be IgG-positive $(P=0.64)$. The seroprevalence of $T$. gondii infection was not significantly different in T2DM patients with different educational levels $(P=0.21)$. T. gondii seroprevalence was not significantly different among T1DM patients and source of drinking water $(P=0.292)$ and consumption of raw/ undercooked meat $(P=0.384)$. Among risk factors, only contact with cats was significantly associated with IgG seroprevalence in both T2DM patients $(P<0.001)$ and control subjects $(P=0.045)$ (Table 2$)$.

\section{Discussion}

The possible association between toxoplasmosis and DM is still controversial, as there are several studies with conflicting results $[11,13,15-17]$. Since there is a lack of knowledge about the epidemiological status of $T$. gondii infection and its association with T1DM and T2DM in southwest Iran (Khuzestan province, Khorramshahr city), anti-T. gondii IgG antibody in diabetic patients compared to nondiabetic subjects was evaluated. Our findings showed higher seroprevalence of anti-T. gondii IgG antibody in 


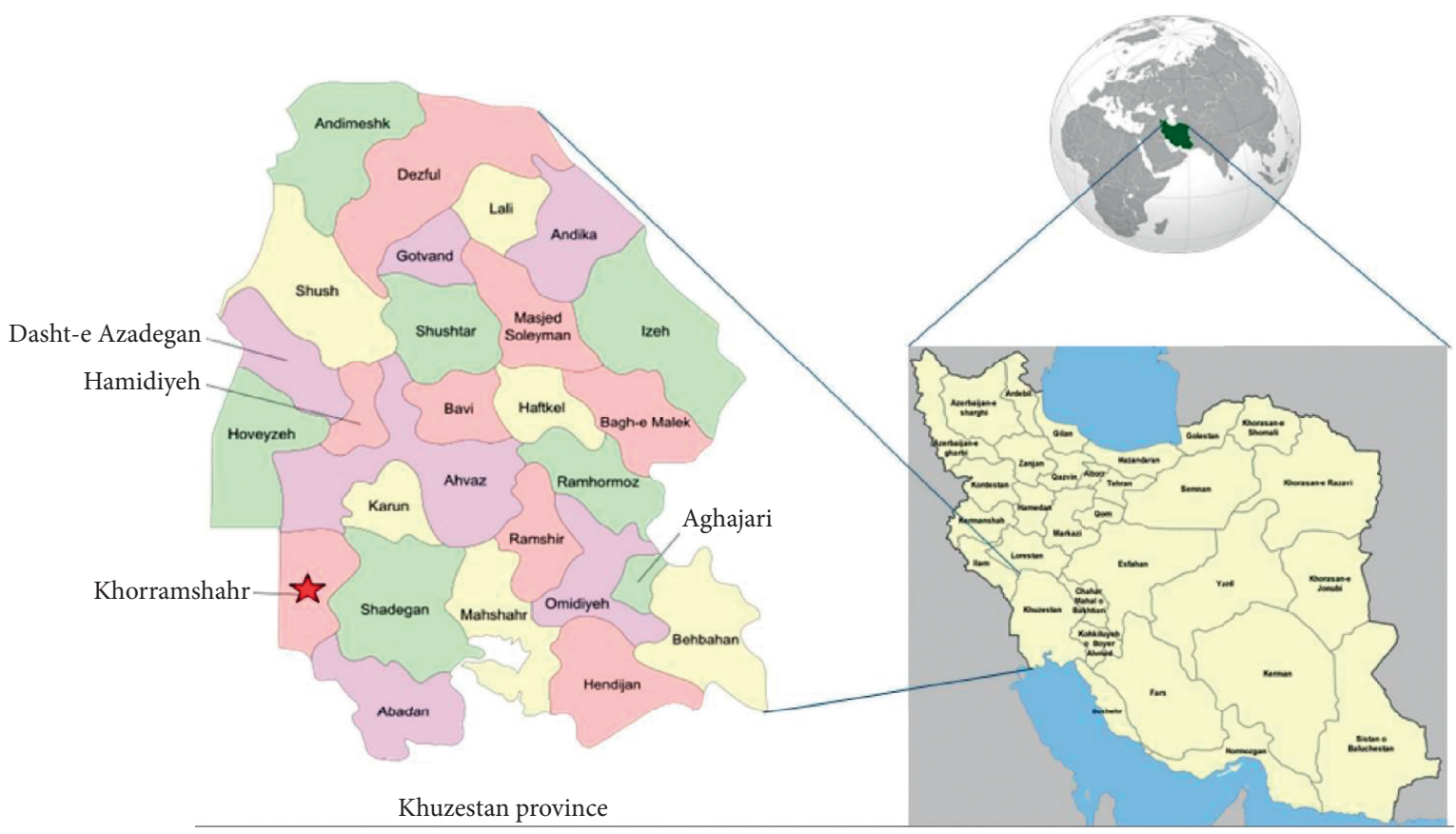

FIGURE 1: Location of Khorramshahr city. The study region is shown with red asterisk.

TABLE 1: Demographic characteristics and risk factors related to the seroprevalence of T. gondii infection in T1DM patients, Khorramshahr city.

\begin{tabular}{|c|c|c|c|c|c|c|c|c|c|}
\hline \multirow{2}{*}{ Characteristic } & \multicolumn{4}{|c|}{ Type 1 DM $(N=36)$} & \multicolumn{4}{|c|}{ Controls $(N=50)$} & \multirow{2}{*}{$\begin{array}{c}\text { Type } 1 \text { DM versus } \\
\text { controls } \\
P \text { value }\end{array}$} \\
\hline & $\begin{array}{l}\text { No. of } \\
\text { tested }\end{array}$ & IgG-positive & $\%$ & $P$ value & $\begin{array}{l}\text { No. of } \\
\text { tested }\end{array}$ & IgG-positive & $\%$ & $P$ value & \\
\hline \multicolumn{10}{|l|}{ Age } \\
\hline $0-10$ & 5 & 3 & 60.00 & \multirow{4}{*}{0.847} & 8 & 2 & 25.00 & \multirow{4}{*}{0.911} & 0.249 \\
\hline $11-20$ & 9 & 6 & 66.66 & & 12 & 4 & 33.33 & & 0.142 \\
\hline $21-30$ & 10 & 8 & 80.00 & & 15 & 5 & 33.33 & & 0.029 \\
\hline $31-40$ & 12 & 8 & 66.66 & & 15 & 6 & 40.00 & & 0.161 \\
\hline \multicolumn{10}{|l|}{ Gender } \\
\hline Female & 17 & 14 & 82.35 & \multirow{2}{*}{0.109} & 25 & 9 & 36.00 & \multirow{2}{*}{0.765} & 0.003 \\
\hline Male & 19 & 11 & 57.89 & & 25 & 8 & 32.00 & & 0.086 \\
\hline \multicolumn{10}{|l|}{ Residence } \\
\hline Urban & 25 & 16 & 64.00 & \multirow{2}{*}{0.254} & 30 & 10 & 33.33 & \multirow[t]{2}{*}{0.903} & 0.023 \\
\hline Rural & 11 & 9 & 81.81 & & 20 & 7 & 35.00 & & 0.016 \\
\hline \multicolumn{10}{|l|}{ Education level } \\
\hline Diploma or lower & 27 & 20 & 74.07 & \multirow{2}{*}{0.261} & 35 & 12 & 34.28 & \multirow{2}{*}{0.948} & 0.002 \\
\hline University degree & 9 & 5 & 55.55 & & 15 & 5 & 33.33 & & 0.26 \\
\hline \multicolumn{10}{|l|}{ Contact with cat } \\
\hline Yes & 24 & 19 & 79.16 & \multirow{2}{*}{0.073} & 36 & 13 & 36.11 & \multirow{2}{*}{0.438} & 0.002 \\
\hline No & 12 & 6 & 50.00 & & 14 & 4 & 28.57 & & 0.237 \\
\hline \multicolumn{10}{|c|}{ Source of drinking water } \\
\hline Unpurified water & 9 & 6 & 66.66 & \multirow{2}{*}{0.571} & 9 & 4 & 44.44 & \multirow{2}{*}{0.358} & 0.319 \\
\hline Purified water & 27 & 19 & 70.37 & & 41 & 13 & 31.70 & & 0.002 \\
\hline \multicolumn{10}{|l|}{$\begin{array}{l}\text { Consumption of raw/ } \\
\text { undercooked meat }\end{array}$} \\
\hline Yes & 6 & 4 & 66.66 & \multirow{2}{*}{0.609} & 10 & 5 & 50.00 & \multirow{2}{*}{0.204} & 0.451 \\
\hline No & 30 & 21 & 70.00 & & 40 & 12 & 30.00 & & 0.001 \\
\hline Total & 36 & 25 & 69.4 & & 50 & 17 & 34.0 & & 0.001 \\
\hline
\end{tabular}


TABLE 2: Demographic characteristics and risk factors related to the seroprevalence of T. gondii infection in T2DM patients, Khorramshahr city.

\begin{tabular}{|c|c|c|c|c|c|c|c|c|c|}
\hline \multirow{2}{*}{ Characteristic } & \multicolumn{4}{|c|}{ Type 2 DM $(N=69)$} & \multicolumn{4}{|c|}{ Controls $(N=100)$} & \multirow{2}{*}{$\begin{array}{c}\text { Type } 2 \text { DM versus } \\
\text { controls } \\
P \text { value }\end{array}$} \\
\hline & $\begin{array}{l}\text { No. of } \\
\text { tested }\end{array}$ & IgG-positive & $\%$ & $P$ value & $\begin{array}{l}\text { No. of } \\
\text { tested }\end{array}$ & IgG-positive & $\%$ & $\begin{array}{c}P \\
\text { value }\end{array}$ & \\
\hline \multicolumn{10}{|l|}{ Age } \\
\hline$\leq 40$ & 12 & 8 & 66.66 & \multirow{3}{*}{0.884} & 25 & 7 & 28.00 & \multirow{3}{*}{0.93} & 0.03 \\
\hline $41-60$ & 34 & 25 & 73.52 & & 50 & 14 & 28.00 & & $<0.001$ \\
\hline$>60$ & 23 & 17 & 73.91 & & 25 & 8 & 32.00 & & 0.004 \\
\hline \multicolumn{10}{|l|}{ Gender } \\
\hline Female & 35 & 27 & 77.14 & \multirow{2}{*}{0.377} & 50 & 13 & 26.00 & \multirow{2}{*}{0.509} & $<0.001$ \\
\hline Male & 34 & 23 & 67.64 & & 50 & 16 & 32.00 & & 0.001 \\
\hline \multicolumn{10}{|l|}{ Residence } \\
\hline Urban & 43 & 32 & 74.41 & \multirow{2}{*}{0.64} & 60 & 19 & 31.66 & \multirow{2}{*}{0.472} & $<0.001$ \\
\hline Rural & 26 & 18 & 69.23 & & 40 & 10 & 25.00 & & $<0.001$ \\
\hline \multicolumn{10}{|l|}{ Education level } \\
\hline Diploma or lower & 51 & 39 & 76.47 & \multirow{2}{*}{0.21} & 66 & 21 & 31.81 & \multirow{2}{*}{0.387} & $<0.001$ \\
\hline University degree & 18 & 11 & 61.11 & & 34 & 8 & 23.52 & & 0.007 \\
\hline \multicolumn{10}{|l|}{ Contact with cat } \\
\hline Yes & 52 & 44 & 84.51 & \multirow{2}{*}{$<0.001$} & 73 & 25 & 34.24 & \multirow{2}{*}{0.045} & $<0.001$ \\
\hline No & 17 & 6 & 35.29 & & 27 & 4 & 14.81 & & 0.114 \\
\hline \multicolumn{10}{|c|}{ Source of drinking water } \\
\hline Unpurified water & 7 & 4 & 57.14 & \multirow{2}{*}{0.292} & 11 & 6 & 54.54 & \multirow{2}{*}{0.048} & 0.648 \\
\hline Purified water & 62 & 46 & 74.19 & & 89 & 23 & 25.84 & & $<0.001$ \\
\hline \multicolumn{10}{|l|}{$\begin{array}{l}\text { Consumption of raw/ } \\
\text { undercooked meat }\end{array}$} \\
\hline Yes & 8 & 5 & 62.50 & \multirow{2}{*}{0.384} & 14 & 7 & 50.00 & \multirow{2}{*}{0.062} & 0.454 \\
\hline No & 61 & 45 & 73.77 & & 86 & 22 & 25.58 & & $<0.001$ \\
\hline Total & 69 & 50 & 72.5 & & 100 & 29 & 29.00 & & $<0.001$ \\
\hline
\end{tabular}

T1DM and T2DM patients in comparison to nondiabetic individuals. Thus, the results of our study based on ELISA method supported the association between chronic toxoplasmosis and both types of DM.

T1DM is considered as an autoimmune disease, which is probably associated with genetic and environmental factors [10]. The association between infectious agents and T1DM has been approved $[18,19]$. In this study, higher seroprevalence of T. gondii infection in T1DM patients in comparison to nondiabetic individuals was observed $(69.4 \%$ versus $34.0 \%)$. T. gondii can infect all nucleated cells, including pancreatic $\beta$-cells. Pancreas can secret insulin, which is crucial for controlling blood glucose level. Any deficiency in insulin production may cause the occurrence of T1DM. Therefore, T. gondii infection could develop T1DM $[12,16,20]$. In the other hand, the diabetic patients are considered as immunocompromised subjects and are more vulnerable to infection with $T$. gondii than healthy individuals [21].

$\mathrm{T} 2 \mathrm{DM}$ is a metabolic disease and, as a major global health concern, its incidence rate has increased during the recent decade throughout the globe $[10,22]$. In the current study, $72.5 \%$ of T2DM patients and $29.0 \%$ of nondiabetic subjects were seropositive for anti-T. gondii IgG antibody, and the difference was statistically significant $(P<0.001)$. The same results were reported by Ozcelik et al. from Turkey [23]. In contrast with the results of our study, Molan et al. reported that $62.0 \%$ and $66.0 \%$ of the T2DM patients and nondiabetic subjects were seropositive for T.gondii infection, respectively, but the difference was not statistically significant [17]. In a review paper with meta-analysis approach, Majidiani et al. reviewed seven articles to investigate the association between T. gondii infection and DM from a global perspective. They concluded that latent toxoplasmosis accounts as a possible risk factor for T2DM (OR: 2.39; 95\% CI: $1.20-4.75 ; P=0.013)$, while no statistically significant association was observed between T. gondii and T1DM (OR: 1.10; 95\% CI: 0.13-9.57; $P=0.929$ ) [13]. The discordance between studies could be explained due to the study area, the number of participants in the case and control groups, different type of sampling, environmental factors, lifestyle and habits of the people as well as different specificity and sensitivity of the laboratory techniques, variable cutoff values, or antibody titers for serological kits.

In the current research, the main risk factors of $T$. gondii infection were assessed. A significant association between T. gondii seroprevalence and contact with cats was found in both T2DM patients and nondiabetic subjects. In the previous studies among general population and patients undergoing hemodialysis, the same results were observed in southwest Iran $[24,25]$. Since cats are considered as the only definitive hosts and are one of the major sources of T. gondii, it seems that close contact with cats is considered as an important risk factor for acquiring the infection. The cats can release several millions of oocysts into the environment and public places through feces $[1,26]$. In addition, the 
sporulated oocysts have the ability to survive for a long time in the optimum conditions in the soil [5]. Based on a review paper, the prevalence of $T$. gondii oocysts was estimated to be $16 \%$ (95\% CI: 10-26) in the soil of public places worldwide [5].

Choosing the appropriate inclusion and exclusion criteria, investigating the clinical and diagnostic history of all the participants, assessment of demographic information and the main risk factors of T. gondii infection through a structured questionnaire, and investigation of both T1DM and T2DM are strengths of the current study. Nonetheless, there are limitations that should be kept in mind: (1) this study was based on sampling from a small number of T1DM and T2DM patients in a limited area; (2) only serological assay by ELISA was performed on samples with no supporting data by molecular confirmation.

\section{Conclusion}

In conclusion, we found high rates of $T$. gondii seroprevalence in diabetic patients in southwest Iran. Although this study revealed a significant association between chronic T. gondii infection and two types of diabetes mellitus (T1DM and $\mathrm{T} 2 \mathrm{DM}$ ), there remain many questions regarding the exact mechanisms of $T$. gondii in the pathogenesis of DM. More studies are required to elucidate the exact association between T. gondii and DM.

\section{Abbreviations}

$\begin{array}{ll}\text { CI: } & \text { Confidence interval } \\ \text { DM: } & \text { Diabetes mellitus } \\ \text { ELISA: } & \text { Enzyme-linked immunosorbent assay } \\ \text { IgG: } & \text { Immunoglobulin G } \\ \text { OR: } & \text { Odds ratio } \\ \text { T. gondii: } & \text { Toxoplasma gondii } \\ \text { T1DM: } & \text { Type 1 diabetes mellitus } \\ \text { T2DM: } & \text { Type } 2 \text { diabetes mellitus. }\end{array}$

\section{Data Availability}

The datasets used and/or analysed during the current study are available from the corresponding author upon reasonable request.

\section{Ethical Approval}

This study received the approval from the Behbahan Faculty of Medical Sciences Ethical Committee (IR.BHN.REC.1399.008).

\section{Consent}

All subjects voluntarily agreed to be tested. A written informed consent was obtained from adult persons and parents or guardians of subjects below 18 years of age.

\section{Conflicts of Interest}

The authors declare that there are no conflicts of interest.

\section{Authors' Contributions}

Shahrzad Soltani, Masoud Foroutan, and Sanaz Tavakoli conceived, designed, and drafted the manuscript; Mohamad Sabaghan, Mehdi Sagha Kahvaz, and Marzieh Pashmforosh contributed to data acquisition; Shahrzad Soltani and Masoud Foroutan contributed to statistical analysis; Masoud Foroutan critically revised the text. All authors read and approved the final version of the manuscript. The corresponding authors had access to the data in the study and had final responsibility for the decision to submit for publication

\section{Acknowledgments}

The authors sincerely appreciate the kind cooperation of all the personnel at Valiasr Hospital in Khorramshahr city. The authors are very grateful to Mrs. Fatemeh Maghsoudi (Abadan Faculty of Medical Sciences, Abadan, Iran) and Dr. Ali Dalir Ghaffari (Department of Parasitology, Faculty of Medical Sciences, Tarbiat Modares University, Tehran, Iran) for their helpful consultation and comments on the manuscript. This study was financially supported by the Behbahan Faculty of Medical Sciences, Behbahan, Iran (Grant no. 98114). The funders of this study had no role in the study design, analysis and interpretation of data, writing of the final paper, and the decision to submit the manuscript for publication.

\section{References}

[1] J. P. Dubey, "The history of Toxoplasma gondii-the first 100 years," The Journal of Eukaryotic Microbiology, vol. 55, pp. 467-475, 2008.

[2] M. Foroutan-Rad, H. Majidiani, S. Dalvand et al., "Toxoplasmosis in blood donors: a systematic review and metaanalysis," Transfusion Medicine Reviews, vol. 30, pp. 116-122, 2016.

[3] S. Belluco, G. Simonato, M. Mancin et al., "Toxoplasma gondii infection and food consumption: a systematic review and meta-analysis of case-controlled studies," Critical Reviews in Food Science and Nutrition, vol. 58, pp. 3085-3096, 2018.

[4] S. Fallahi, A. Rostami, M. Nourollahpour Shiadeh et al., "An updated literature review on maternal-fetal and reproductive disorders of Toxoplasma gondii infection," Journal of Gynecology Obstetrics and Human Reproduction, vol. 47, pp. 133140, 2018.

[5] B. Maleki, N. Ahmadi, M. Olfatifar et al., "Toxoplasma oocysts in the soil of public places worldwide: a systematic review and meta-analysis," Transactions of the Royal Society of Tropical Medicine and Hygiene, 2020.

[6] Z. D. Wang, H. H. Liu, Z. X. Ma et al., "Toxoplasma gondii infection in immunocompromised patients: a systematic review and meta-analysis," Frontiers in Microbiology, vol. 8, p. 389, 2017.

[7] A. Rostami, S. M. Riahi, H. R. Gamble et al., "Global prevalence of latent toxoplasmosis in pregnant women: a systematic review and meta-analysis," Clinical Microbiology and Infection, vol. 26, pp. 673-683, 2020.

[8] M. Foroutan, A. Rostami, H. Majidiani et al., "A systematic review and meta-analysis of the prevalence of toxoplasmosis in hemodialysis patients in Iran," Epidemiology and Health, vol. 40, Article ID e2018016, 2018. 
[9] M. Foroutan, S. Dalvand, A. Daryani et al., "Rolling up the pieces of a puzzle: a systematic review and meta-analysis of the prevalence of toxoplasmosis in Iran," Alexandria Journal of Medicine, vol. 54, pp. 189-196, 2018.

[10] S. Canivell and R. Gomis, "Diagnosis and classification of autoimmune diabetes mellitus," Autoimmunity Reviews, vol. 13, pp. 403-407, 2014.

[11] K. Nosaka, M. Hunter, and W. Wang, "The role of Toxoplasma gondii as a possible inflammatory agent in the pathogenesis of type 2 diabetes mellitus in humans," Family Medicine and Community Health, vol. 4, pp. 44-62, 2016.

[12] J. T. Prandota, "Gondii infection acquired during pregnancy and/or after birth may be responsible for development of both type 1 and 2 diabetes mellitus," Journal of Diabetes \& Metabolism, vol. 4, p. 55, 2013.

[13] H. Majidiani, S. Dalvand, A. Daryani et al., "Is chronic toxoplasmosis a risk factor for diabetes mellitus? A systematic review and meta-analysis of case-control studies," The Brazilian Journal of Infectious Diseases, vol. 20, pp. 605-609, 2016.

[14] WHO, Definition, Diagnosis and Classification of Diabetes Mellitus and its Complications: Report of a WHO Consultation. Part 1, Diagnosis and Classification of Diabetes Mellitus, World Health Organization, Geneva, Switzerland, 1999.

[15] C. Alvarado-Esquivel, N. Loera-Moncivais, J. HernandezTinoco et al., "Lack of association between Toxoplasma gondii infection and diabetes mellitus: a matched case-control study in a Mexican population," Journal of Clinical Medicine Research, vol. 9, pp. 508-511, 2017.

[16] E. V. Nassief Beshay, S. A. El-Refai, M. A. Helwa et al., "Toxoplasma gondii as a possible causative pathogen of type-1 diabetes mellitus: evidence from case-control and experimental studies," Experimental Parasitology, vol. 188, pp. 93101, 2018.

[17] A. Molan, K. Nosaka, M. Hunter et al., "First age- and gendermatched case-control study in Australia examining the possible association between Toxoplasma gondii infection and type 2 diabetes mellitus: the busselton health study," Journal of Parasitology Research, vol. 2020, Article ID 3142918, 2020.

[18] K. M. Drescher, M. von Herrath, and S. Tracy, "Enteroviruses, hygiene and type 1 diabetes: toward a preventive vaccine," Reviews in Medical Virology, vol. 25, pp. 19-32, 2015.

[19] C. M. Filippi and M. G. von Herrath, "Viral trigger for type 1 diabetes: pros and cons," Diabetes, vol. 57, pp. 2863-2871, 2008.

[20] S. Zhu, D. H. Lai, S. Q. Li et al., "Stimulative effects of insulin on Toxoplasma gondii replication in 3T3-L1 cells," Cell Biology International, vol. 30, pp. 149-153, 2006.

[21] J. Saki, S. Shafieenia, and M. Foroutan-Rad, "Seroprevalence of toxoplasmosis in diabetic pregnant women in southwestern of Iran," Journal of Parasitic Diseases, vol. 40, pp. 1586-1589, 2016.

[22] G. B. D. Diseases and C. Injuries, "Global burden of 369 diseases and injuries in 204 countries and territories, 1990-2019: a systematic analysis for the Global burden of disease study 2019," Lancet, vol. 396, pp. 1204-1222, 2020.

[23] S. Ozcelik, M. Alim, and N. Ozpinar, "Detection of toxoplasma gondii infection among diabetic patients in Turkey," Clinical Epidemiology and Global Health, vol. 8, no. 3, pp. 899-902, 2020.

[24] S. Soltani, M. S. Kahvaz, S. Soltani et al., "Seroprevalence and associated risk factors of Toxoplasma gondii infection in patients undergoing hemodialysis and healthy group," $B M C$ Research Notes, vol. 13, p. 551, 2020.
[25] S. Soltani, M. Foroutan, H. Afshari et al., "Seroepidemiological evaluation of Toxoplasma gondii immunity among the general population in southwest of Iran," Journal of Parasitic Diseases, vol. 42, pp. 636-642, 2018.

[26] J. P. Dubey, C. K. Cerqueira-Cezar, F. H. A. Murata et al., “All about toxoplasmosis in cats: the last decade," Veterinary Parasitology, vol. 283, Article ID 109145, 2020. 